Retos, I0(19), 2020

\title{
Crecimiento económico en una región emprendedora en el Ecuador
}

\section{Economic growth in an enterprising region in Ecuador}

\begin{abstract}
Paúl Moina-Sánchez es investigador de la Universidad Técnica de Ambato (Ecuador) (pmoina9124@uta.edu.ec)
\end{abstract} (https://orcid.org/0000-0001-5286-6787)

Lilián Morales-Carrasco es docente-Investigadora de la Universidad Técnica de Ambato (Ecuador) (lilianmorales@uta.edu.ec) (http://orcid.org/0000-0001-7026-4544)

Ana Córdova-Pacheco es docente-investigadora de la Universidad Técnica de Ambato (Ecuador) (anaccordova@uta.edu.ec) (https://orcid.org/0000-0001-6330-3306)

\section{Resumen}

Este artículo analiza los niveles de emprendimiento temprano de las industrias estratégicas del principal puerto del Ecuador, en la provincia de Guayas en el período 2012-2016. Se utilizaron fuentes secundarias en lo que respecta a la actividad empresarial correspondientes a las sociedades en la Superintendencia de Compañías del Ecuador y del Servicio de Rentas Internas del Ecuador para las empresas unipersonales, por otro lado del Banco Central del Ecuador se obtuvieron datos del Valor Agregado Bruto y la población económicamente activa, la tasa de desempleo del Instituto de Estadísticas y Censos del Ecuador; con esta información se obtiene la TEA (Tasa de actividad emprendedora temprana) y el porcentaje de las empresas establecidas en la provincia de Guayas. Se diseñaron dos modelos econométricos mediante mínimos cuadrados ordinarios, en los cuales se comprobó la incidencia de la TEA y las empresas establecidas en el crecimiento económico y la incidencia del desempleo y las empresas establecidas en la TEA. Los resultados evidenciaron que la TEA y la Tasa de Empresas Establecidas explican en un 97,07\% el crecimiento económico de la provincia especialmente en los sectores textiles, confecciones, alimentos y bebidas procesadas donde se evidencian altas tasas de entrada nacientes y nuevas, además se constató que existe una relación inversa entre el desempleo y el emprendimiento.

\begin{abstract}
This article analyzes the levels of early entrepreneurship of the strategic industries of the main port of Ecuador, in the province of Guayas in the period 2012-2016. Secondary sources were used in relation to the business activity corresponding to the companies in the Superintendence of Companies of Ecuador and the Internal Revenue Service of Ecuador for sole proprietorships, on the other hand, the Central Bank of Ecuador, Value Added Data was obtained. Gross and the economically active population, the unemployment rate of the Institute of Statistics and Census of Ecuador; with this information we obtain the TEA (rate of early entrepreneurial activity) and the\% of companies established in the province of Guayas, to then design two econometric models by ordinary least squares, in which the incidence of ASD was verified and established companies in economic growth and the incidence of unemployment and companies established in the TEA. The results showed that the TEA and the rate of established companies accounted for $97.07 \%$ of the province's economic growth, especially in the textile, garment, processed foods and beverages sectors, where high new and emerging entry rates are evident. found that there is an inverse relationship between unemployment and entrepreneurship.
\end{abstract}

\section{Palabras clave I keywords}

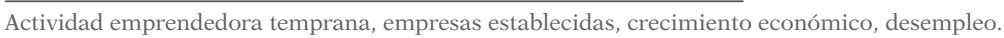
Early entrepreneurial activity, established companies, economic growth, unemployment.

Cómo citar: Moina-Sánchez, P., Morales-Carrasco, L., y Córdova-Pacheco, A. (2020). Crecimiento económico en una región emprendedora en el Ecuador. Retos Revista de Ciencias de la Administración y Economía, 10(19), 65-80. https://doi.org/10.17163/ret.n19.2020.04 


\section{Introducción}

El emprendimiento es un proceso global presente en todos los países, que se ha desarrollado desde la existencia del hombre, el cual ha explorado y trabajado para procurarse siempre un sustento. Esta iniciativa económica es vista como una opción a la incertidumbre laboral y a la necesidad de generación de riqueza (Rodríguez, 2014). Según el reporte del Global Entrepreneurship Monitor —GEM-(2017), en la última década y media los países de América Latina y el Caribe han presentado cambios significativos, principalmente positivos. Cambios promovidos por políticas macroeconomías prudentes que les han permitido afrontar choques externos, además los niveles de pobreza se redujeron a menos de la mitad de lo que fueron a inicios del 2000, la clase media ha crecido, y se ha hecho una fuerte inversión en educación, infraestructura y fortalecimiento productivo de las empresas.

El emprendimiento en América Latina y el Caribe - ALC - es abundante, sin embargo, de acuerdo al reporte del Banco Mundial (2014) las empresas en esta región son más pequeñas y menos proclives a crecer e innovar. El informe revela algunos hechos positivos, ALC es una zona de emprendedores donde el número de empresas per cápita es mayor que en otros países y regiones y en la cual inesperadamente la tasa de emprendedores cuyos negocios están legalmente registrados es considerablemente alto en varias economías de la región. No obstante, pese a existir un gran número de empresas, hay una considerable diferencia en concepto de innovación entre ALC y el resto de las regiones. Este atraso afecta a empresas grandes y pequeñas por igual, incluyendo las empresas con mercado exportador.

En Ecuador el término emprendimiento comienza a ganar fuerza desde el año 2000 (Araque, 2015). Ecuador es el país con la actividad emprendedora temprana - TEA - más alta de América Latina y el Caribe, pues según el reporte del GEM Ecuador 2016 (2017) la tasa de Actividad Emprendedora Temprana al año 2016 fue de $31.8 \%$, el porcentaje más alto se registró en el año 2013 con un 35,9\% denominado "el boom del emprendimiento en el Ecuador".

En este contexto, las cuestiones que intenta resolver este artículo son: ¿El crecimiento económico de la provincia del Guayas se debe a la TEA y los negocios establecidos? ¿Los índices de desempleo de la provincia guardan relación con la actividad emprendedora?

El artículo presenta la siguiente estructura: en la primera parte se hace una breve revisión de la literatura sobre el emprendimiento y el crecimiento económico; en la segunda parte se presenta la metodología que se utiliza para la estimación de los diferentes datos y modelos econométricos; la tercera parte indica los resultados obtenidos; la cuarta parte presenta la discusión de resultados; finalmente, se muestran las conclusiones obtenidas de la investigación.

\subsection{Revisión de la literatura}

La relación entre emprendimiento y crecimiento económico ha sido ampliamente estudiada, los resultados inferidos en las distintas investigaciones no indican un único patrón, si no que varían en función de la región analizada y las variables e indicadores utilizados. Sin embargo, la mayoría de estudios confirman la relación 
positiva entre la actividad emprendedora y el crecimiento económico (Stam, Suddle, Hessels, \& Stel, 2006; Acs, Audretsch, Braunerhjelm \& Carlsson, 2012; Galindo \& Méndez, 2012; Dau \& Cuervo-Cazurra, 2014; Galindo, Méndez, \& Castaño, 2016).

Lupiáñez, Priede y López (2014) y Galindo et al. (2016) llevan a cabo una revisión de un número importante de investigaciones que abordan esta relación, donde se destaca que el fomento de una base empresarial sólida genera riqueza, empleo y bienestar (Comisión Europea, 2003), a su vez el estudio de Lupiáñez et al. (2014) plantea que el conocimiento es un factor decisivo, pues el emprendedor es el agente económico con la habilidad de convertir conocimiento, en conocimiento con valor económico, siendo una figura indispensable en el crecimiento económico.

Para poder comprender la relación entre la actividad emprendedora y el crecimiento económico en algunos estudios se toma en cuenta el papel que desempeñan las variables culturales (Jaén, Fernández \& Liñán, 2013; Pinillos Costa, 2011), la innovación e inversión privada (Galindo \& Méndez, 2012) y la inversión en conocimientos (Acs, Audretsch, Braunerhjelm \& Carlsson, 2012), variables determinantes en dichos trabajos. En la investigación de Jaén et al. (2013) la variable actividad emprendedora temprana conjuntamente con las variables culturales ayudan a predecir el nivel de crecimiento económico, dicha relación se contrastó utilizando un análisis de regresión lineal sobre una muestra de 56 países (2001 al 2011), el cual reveló una relación global negativa entre la TEA y el nivel de ingresos per cápita en la que la actividad emprendedora explica en un 37,8\% la varianza del nivel de ingresos.

Galindo y Méndez (2012) para determinar la relación entre emprendedores y crecimiento económico consideran una muestra de 11 países ${ }^{1}$ durante el periodo 2000-2010, para ello utilizaron un modelo de regresión logarítmica, el cual arrojó resultados significativos donde los emprendedores ejercen un efecto positivo sobre el crecimiento, siendo ésta la principal diferencia con el estudio de Jaén et al. (2013) que identificó una relación inversa entre estos dos factores. Cabe destacar que en Galindo y Méndez (2012) también incluyeron en el modelo de regresión las variables exógenas, innovación e inversión privada, en el que la innovación también explica positivamente el crecimiento económico, conjuntamente con la actividad emprendedora. Posteriormente Galindo, Méndez, y Castaño (2016) amplían esta perspectiva, en su investigación afirman que el emprendimiento es uno de los principales factores para estimular el crecimiento económico, pues el emprendedor es quien utiliza los recursos de capital y trabajo, para la generación de riqueza que se distribuirá en la sociedad, es primordial que esta distribución sea justa, evitando tensiones sociales que afecten la conducta y expectativas del emprendedor. Por ello para una correcta justicia distributiva, que se centra en que cada uno reciba según su contribución, el papel del gobierno es esencial a través de políticas económicas y principios de compensación que generen un equilibrio en el clima social, que induzca a los empresarios a generar riqueza. Además señalan que crecimiento económico no es un símil de progreso económico, en concordancia con el trabajo de Holcombe (2007 citado en Galindo, Méndez y Castaño, 2016) quien indica que el progreso está relacionado con la calidad del producto mientras que el crecimiento lo está con la cantidad, siendo el

1 España, Dinamarca, Alemania, Francia, Italia, Finlandia, Holanda, Reino Unido, Suecia, Japón y Estados Unidos. 
emprendedor un factor esencial en ambos procesos, ya que este mismo agente económico es el encargado de introducir la innovación en el proceso productivo, factor que incide en el progreso, por lo que el emprendimiento también es el principal factor del progreso económico.

Por su parte Acs et al. (2012) mediante un modelo econométrico de mínimos cuadrados generalizados determinaron si la actividad emprendedora tiene un impacto positivo y sistemático en el crecimiento económico en un total de 18 países del año 1981 al 1998; estudio que constató que el espíritu empresarial genera una contribución positiva al crecimiento económico. Además, se identificó que la inversión en conocimiento, medida por el porcentaje de PIB en I+D y los años de escolaridad de la población, son una fuente importante de crecimiento económico. Siguiendo ésta línea de investigación Stam, Suddle, Hessels, y Stel (2006) no solo determinan la relación entre el emprendimiento y el crecimiento económico, sino también en averiguar qué tipo de empresario influye más en dicho crecimiento. En dicha investigación se hizo una diferencia de la Actividad Emprendedora Total en TEA-general, TEA-crecimiento fuerte, quienes esperan emplear 20 trabajadores o más dentro de cinco años y TEAcrecimiento medio, quienes esperan emplear a 6 trabajadores o más dentro de cinco años, datos obtenidos del GEM (2002) para una muestra de 36 países. Además de estas variables, también incluyeron en el modelo a las variables independientes renta per cápita y crecimiento de la competitividad. Para contrastar la incidencia que tienen estos factores en el crecimiento económico, los autores usaron un modelo econométrico de regresión simple, cuyos resultados sustentaron que el espíritu emprendedor ambicioso, es decir propietarios quienes esperan emplear 20 trabajadores o más, contribuyen más al crecimiento económico que la actividad emprendedora en general. También se pudo verificar que esto generalmente sucedía en los países en transición.

Desde otra perspectiva Dau y Cuervo-Cazurra (2014) plantean un enfoque en el que el emprendimiento depende del crecimiento económico y de las instituciones pro-mercado. Para ello distinguen el emprendimiento en formal e informal, en cuanto a las instituciones pro-mercado las separan en sus dos principales componentes, la liberación económica y los niveles de gobernanza. Como resultado de la investigación se constató que el crecimiento económico medido por el crecimiento del PIB presenta un efecto significativo y positivo en ambos tipos de emprendimiento, mientras que los niveles de gobernanza presentan un efecto positivo en el emprendimiento formal, pero negativo en el informal y en el emprendimiento total. La liberación económica tiene un efecto positivo en los dos tipos de emprendimiento. Siguiendo el mismo enfoque pero con distinta conjetura, Liñán, Fernández y Romero (2013) en su investigación plantean una hipótesis en la que la tasa de actividad empresarial se correlaciona negativamente con el PIB per cápita, es decir que un mayor ingreso se asocia con una menor iniciativa empresarial. La muestra de estudio corresponde a un total de 56 países y para comprobar dicha hipótesis se empleó un modelo econométrico de mínimos cuadrados parciales. Los resultados del modelo evidenciaron que el PIB per cápita juega un importante papel para explicar el nivel de actividad emprendedora en los países de la muestra. Tal como se planteó en la hipótesis el nivel de ingresos está relacionado negativamente con la TEA.

Pinillos Costa (2011) estudió las variables que afectan al emprendimiento con un enfoque ligeramente distinto, donde no solo trabajó con variables económicas 
y socio-demográficas sino también con factores culturales, que es la variable independiente de su estudio. La hipótesis base de la investigación consiste en verificar si mientras la cultura de la sociedad es más posmaterialista ${ }^{2}$ más reducida será la tasa de actividad emprendedora total, para contrastar dicha hipótesis se desarrolló una regresión lineal simple donde la variable independiente es el nivel de posmaterialismo y la variable dependiente es la TEA. Además, se realizaron diferentes regresiones múltiples incluyendo variables económicas y socio demográficas, como el desempleo, la renta per cápita y el nivel de educación. Como resultado principal se halló que el posmaterialismo se relaciona negativamente con la actividad emprendedora, llegándose a comprobar la hipótesis de estudio en la cual, las sociedades menos materialistas tienden a presentar valores más bajos de actividad emprendedora temprana. Cabe destacar que en este estudio las variables socioeconómicas, desempleo, renta per cápita o nivel de estudios, no fueron significativas para predecir la actividad emprendedora.

Por último el estudio de Zuniga, Soza, y Soria (2015) que analizaron la incidencia que tiene el desempleo en el emprendimiento, sugiere que la relación entre el desempleo y la actividad emprendedora es difícil de establecer para su zona de investigación, pues únicamente para el periodo 2011-2012 se pudo demostrar que los cambios en el desempleo tuvieron un impacto positivo en el emprendimiento, mientras que para el resto de años no fue posible establecer ninguna clase de relación entre estas dos variables. Su estudio se enfoca las regiones de la Patagonia Chilena durante el periodo 2009-2012, cuyo objetivo principal es contrastar la existencia del efecto oportunidad o efecto refugio, ${ }^{3}$ efectos estudiados en las investigaciones de Reynolds et al. (1995), Blanchflower y Meyer (1994) y Audretsch (1995) citados en Zuniga, Soza, y Soria (2015).

En base a esta literatura se plantean las siguientes hipótesis de la presente investigación.

H1: La actividad emprendedora temprana - TEA — y las empresas establecidas inciden en el crecimiento económico de la provincia de Guayas-Ecuador.

$\mathrm{H} 2$ : El desempleo y las empresas establecidas inciden en la actividad emprendedora temprana - TEA- de la provincia de Guayas-Ecuador.

\section{Materiales y métodos}

\subsection{Datos y variables}

Se utilizaron fuentes de datos secundarias, la información de sociedades provino de la Superintendencia de Compañías Valores y Seguros — SUPERCIAS - mientras que la información de empresas unipersonales se obtuvo del Servicio de Rentas Internas -SRI - por otro lado, la fuente de los datos del Valor Agregado Bruto, fue el Banco Central del Ecuador — BCE — y finalmente, del Instituto Nacional de Estadística y

2 Postmaterialismo es el grado en que una sociedad busca objetivos de vida inmateriales, como el desarrollo personal y la autoestima, sobre la seguridad material, término acuñado por Inglehart en el año 1977.

3 El efecto oportunidad explica una relación inversa entre el nacimiento de nuevas empresas y el desempleo, mientras que el efecto refugio explica que un aumento en el desempleo aumenta el interés por emprender. 
Censos - INEC - se consiguieron los datos de la población económicamente activa y de la Tasa de Desempleo.

El estudio se ejecutó para el periodo 2012-2016 con información de la provincia de Guayas, que es considerada uno de los polos de desarrollo económico en Ecuador y se analizaron los sectores industriales estratégicos según lo establecido por la Secretaría Nacional de Planificación y Desarrollo — SENPLADES - en la agenda Zonal 8, a saber, astillería, siderúrgica, farmacéutica, metalmecánica, tejidos y confecciones, alimentos y bebidas y desarrollo de hardware y software.

Para el presente estudio se consideraron tanto empresas unipersonales como sociedades de las industrias mencionadas, así mismo para facilitar la identificación y clasificación de las empresas por tipo de industria se recurrió al uso de la Clasificación Industrial Internacional Uniforme - CIIU - adoptado por el INEC en la Clasificación Nacional de Actividades Económicas, lo que permitió la depuración y conteo del número de empresas.

La Actividad Emprendedora Temprana - TEA - y las empresas establecidas, se identifican en base a las conceptualizaciones utilizadas por el Global Entrepreneurship Monitor -GEM-.

Para el GEM, la TEA es el porcentaje de la población adulta que está involucrada activamente en emprendimientos nacientes (empresas con no más de 3 meses de existencia) o emprendimientos nuevos (empresas de más de 3 meses, pero no más de 42 meses).

Rocha (2013) calcula la TEA por año utilizando la fórmula:

$$
T E A=\frac{\text { Enacientes }+ \text { Enuevos }}{\text { PEA Guayas }} * 100
$$

Dónde Enacientes es el número total de emprendimientos nacientes de los sectores estratégicos industriales de estudio, Enuevos corresponde al número total de emprendimientos nuevos de los sectores estratégicos industriales en estudio y la PEA Guayas que es la población activa de la provincia.

Las empresas establecidas, se calculan como un porcentaje de la población adulta que está involucrada activamente en un negocio con más de 42 meses.

Se adaptó la fórmula de la TEA, para cuantificar las empresas establecidas.

$$
\text { Establecidas }=\frac{\text { Nestablecidas }}{\text { PEA Guayas }} * 100
$$

Dónde Nestablecidas hace referencia al número total de empresas establecidas de los sectores estratégicos industriales en estudio, mientras que la PEA Guayas es la población activa de la provincia.

La variable crecimiento económico tiene como indicador al Valor Agregado Bruto - VAB — de la provincia de Guayas, cuya información se obtuvo de las Cuentas Provinciales del portal de información del BCE. La variable Tasa de Desempleo, hace referencia a la situación del ciudadano que carece de empleo en el mercado laboral, cuyas tasas han sido tomadas del INEC para la provincia de Guayas. 
Tabla 1. Variables, indicadores y fuentes de información

\begin{tabular}{|l|l|l|}
\hline \multicolumn{1}{|c|}{ Variable } & \multicolumn{1}{|c|}{ Indicador } & \multicolumn{1}{c|}{ Descripción } \\
\hline $\begin{array}{l}\text { Actividad emprendedora } \\
\text { temprana }\end{array}$ & Tasa de TEA & $\begin{array}{l}\text { Desarrollado en esta investigación } \\
\text { usando datos del SRI, SUPER- } \\
\text { CIAS e INEC. }\end{array}$ \\
\hline Empresas establecidas & $\begin{array}{l}\text { Tasa de Empresas } \\
\text { Establecidas }\end{array}$ & $\begin{array}{l}\text { Desarrollado en esta investigación } \\
\text { usando datos del SRI, SUPER- } \\
\text { CIAS e INEC. }\end{array}$ \\
\hline $\begin{array}{l}\text { Crecimiento económico } \\
\text { provincial }\end{array}$ & VAB & $\begin{array}{l}\text { Valor agregado bruto tomado del } \\
\text { Banco Central del Ecuador }\end{array}$ \\
\hline Desempleo & Tasa de Desempleo & $\begin{array}{l}\text { Tasa de Desempleo tomada del } \\
\text { INEC }\end{array}$ \\
\hline
\end{tabular}

Fuente: Elaboración propia

\subsection{Modelos econométricos}

Para contrastar las hipótesis planteadas y determinar si existe una incidencia significativa entre las variables, se establecieron dos modelos econométricos de regresión, cuyas ecuaciones (3) y (4) fueron estimadas mediante el método de mínimos cuadrados ordinarios - MCO—. Se aplicó el contraste de no linealidad únicamente en la ecuación (4) debido a que en este modelo no se aplicaron logaritmos. Así mismo se comprobó si los residuos de cada uno de los dos modelos siguen una distribución normal.

$$
\log V A B=\beta_{0}+\log \beta_{1} T E A+\log \beta_{2} \text { Establecidas }+\mu
$$

La ecuación 3 busca verificar la hipótesis de incidencia de las variables exógenas TEA y tasa de Empresas establecidas, sobre la variable dependiente crecimiento económico. Se optó por analizar esta relación basándose en las investigaciones de Lupiáñez, Priede y López (2014); Jaén, Fernández y Liñán (2013); Galindo y Méndez (2012); Stam, Suddle, Hessels y Stel (2006); Acs, Audretsch, Braunerhjelm y Carlsson (2012) y Galindo, Méndez y Castaño (2016) que han estudiado la relación de incidencia del emprendimiento en el crecimiento económico.

$$
T E A=\beta_{0}+\beta_{1} \text { Desempleo }+\beta_{2} \text { Establecidas }+\mu
$$

Con la ecuación 4 se pretende contrastar la incidencia que tienen las variables independientes Tasa de Desempleo y Tasa de Empresas Establecidas sobre la variable endógena TEA. Se optó por analizar este modelo basándose en la investigación de Zuniga, Soza, y Soria (2015), que establecieron la relación entre el desempleo y el emprendimiento para un año de su periodo de estudio. De igual forma también lo realizó Pinillos Costa (2011) pero con diferentes resultados, pues no halló significancia entre el desempleo y el emprendimiento. 


\section{Resultados}

La figura 1 muestra la evolución de la TEA y de la Tasa de Empresas Establecidas del período 2012-2016. En dichos porcentajes de aporte de la TEA y del total de empresas establecidas se considera como denominador a la población económicamente activa de la provincia de Guayas. En la figura se observa cómo la TEA ha sido superior a las empresas establecidas durante casi todo el periodo de estudio, con la excepción del último año, el 2016. La TEA ha tenido una tendencia positiva hasta el 2014 donde se registra su pico más alto, a partir de este año ha sufrido un retroceso medianamente significativo. Por otro lado, la Tasa de Empresas Establecidas presenta una tendencia positiva durante todo el periodo de estudio, y donde la mayor variación se produce del año 2015 al 2016, debido a la consolidación de las empresas que nacieron en años previos.

\section{Figura 1. TEA y Tasa de Empresas Establecidas en las Industrias Estratégicas del Guayas}

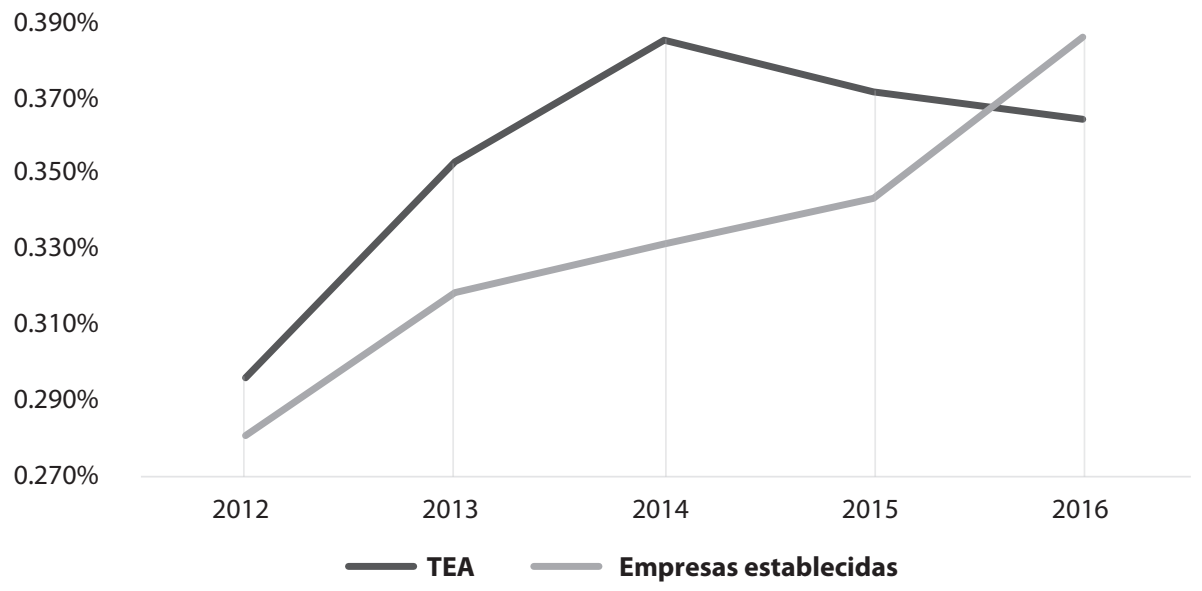

Fuente: Elaboración propia. Base de datos SRI y Superintendencia de Compañías.

Tabla 2. Actividad empresarial total de las Industrias Estratégicas del Guayas

\begin{tabular}{|c|c|c|c|c|c|c|c|}
\hline \multicolumn{2}{|c|}{$\begin{array}{c}\text { Entradas } \\
\text { Brutas }\end{array}$} & $\begin{array}{c}\text { Salidas } \\
\text { Brutas }\end{array}$ & $\begin{array}{c}\text { Entrada } \\
\text { Neta }\end{array}$ & $\begin{array}{c}\text { Turbu- } \\
\text { lencia }\end{array}$ & $\begin{array}{c}\text { Empresas } \\
\text { Nacientes }\end{array}$ & $\begin{array}{c}\text { Empresas } \\
\text { Nuevas }\end{array}$ & $\begin{array}{c}\text { Empresas } \\
\text { Establecidas }\end{array}$ \\
\hline 2012 & 2118 & 985 & 1133 & 3103 & 535 & 4797 & 5050 \\
\hline 2013 & 2138 & 1168 & 970 & 3306 & 557 & 5517 & 5488 \\
\hline 2014 & 2128 & 1575 & 553 & 3703 & 602 & 6121 & 5773 \\
\hline 2015 & 2346 & 1097 & 1249 & 3443 & 609 & 6283 & 6364 \\
\hline 2016 & 2209 & 841 & 1368 & 3050 & 587 & 6388 & 7397 \\
\hline
\end{tabular}

Fuente: Elaboración propia. Base de datos SRI y Superintendencia de Compañías. 


\section{Tabla 3. Empresas Nacientes por Industrias Estratégicas del Guayas}

\begin{tabular}{|c|c|c|c|c|c|c|c|}
\hline \multicolumn{8}{|c|}{ Empresas Nacientes (hasta 3 meses) } \\
\hline & 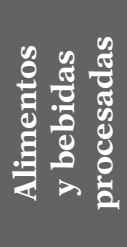 & 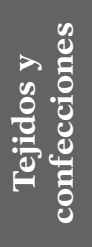 & 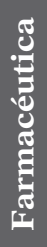 & 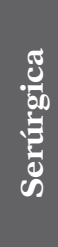 & 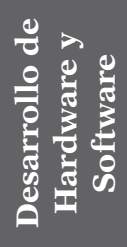 & 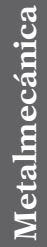 & 茎 \\
\hline 2012 & 117 & 390 & 2 & 10 & 2 & 4 & 10 \\
\hline 2013 & 120 & 407 & 3 & 9 & 3 & 5 & 10 \\
\hline 2014 & 139 & 434 & 0 & 14 & 0 & 4 & 11 \\
\hline 2015 & 195 & 382 & 4 & 10 & 1 & 2 & 15 \\
\hline 2016 & 201 & 361 & 3 & 4 & 0 & 3 & 15 \\
\hline
\end{tabular}

Fuente: Elaboración propia. Base de datos SRI y Superintendencia de Compañías.

Tabla 4. Empresas Nuevas por Industrias Estratégicas del Guayas

\begin{tabular}{|c|c|c|c|c|c|c|c|}
\hline \multicolumn{8}{|c|}{ Empresas Nuevas (más de 3 hasta 42 meses) } \\
\hline & 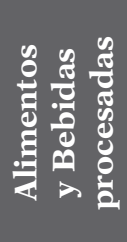 & 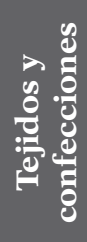 & 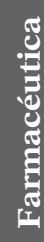 & 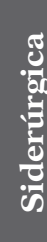 & 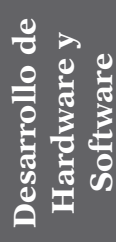 & 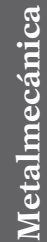 & 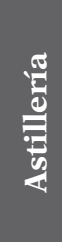 \\
\hline 2012 & 1056 & 3513 & 21 & 66 & 3 & 37 & 101 \\
\hline 2013 & 1195 & 4047 & 22 & 82 & 9 & 37 & 125 \\
\hline 2014 & 1423 & 4404 & 22 & 88 & 16 & 49 & 119 \\
\hline 2015 & 1543 & 4466 & 19 & 76 & 15 & 47 & 117 \\
\hline 2016 & 1863 & 4241 & 22 & 88 & 14 & 41 & 119 \\
\hline
\end{tabular}

Fuente: Elaboración propia. Base de datos SRI y Superintendencia de Compañías. 


\section{Tabla 5. Empresas Establecidas por Industrias Estratégicas del Guayas}

\begin{tabular}{|c|c|c|c|c|c|c|c|}
\hline \multicolumn{8}{|c|}{ Empresas Establecidas (más de 42 meses) } \\
\hline & 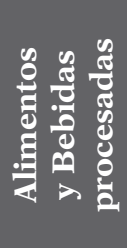 & 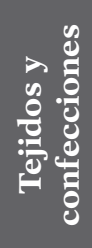 & 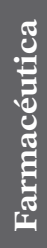 & $\begin{array}{l}\frac{8}{30} \\
\frac{0}{30} \\
\frac{0}{0} \\
\frac{0}{0}\end{array}$ & 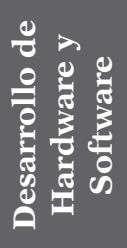 & 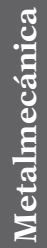 & $\frac{\stackrel{9}{0}}{0}$ \\
\hline 2012 & 1883 & 2708 & 91 & 124 & 7 & 85 & 152 \\
\hline 2013 & 1937 & 3093 & 89 & 113 & 7 & 90 & 159 \\
\hline 2014 & 1922 & 3379 & 88 & 117 & 6 & 90 & 171 \\
\hline 2015 & 1960 & 3952 & 82 & 118 & 7 & 88 & 157 \\
\hline 2016 & 2145 & 4760 & 85 & 128 & 9 & 96 & 174 \\
\hline
\end{tabular}

Fuente: Elaboración propia. Base de datos SRI y Superintendencia de Compañías.

Para el año 2014 se registró el índice más alto de turbulencia, provocado por un aumento de las salidas brutas (Tabla 2), dato relevante pues esto ocurrió un año previo a la desaceleración económica del Ecuador, con lo que se puede apuntar a las empresas como radar de la situación económica, ya que percibieron la contracción del ciclo económico.

Al visualizar la dinámica de la demografía empresarial de los sectores estratégicos del Guayas, se evidenció que la industria de tejidos y confecciones y la de alimentos y bebidas registran el mayor número de empresas tanto en empresas nuevas y nacientes así como las establecidas (Tablas 3, 4 y 5), lo cual fortalece la idea de la especialización de la provincia del Guayas en estos sectores industriales y en menor proporción pero sin duda vigente en el entramado económico, el sector astillero, el más antiguo y tradicional del principal puerto marítimo del Ecuador, acompañado de la siderurgia. Finalmente, sectores como farmacéutica y desarrollo de software y hardware, despuntan con un buen componente de empresas nuevas y establecidas, pero en menor proporción que los anteriores.

\section{Tabla 6. TEA y Tasa de Empresas Establecidas de las Industrias Estratégicas del Guayas}

\begin{tabular}{|c|c|c|c|c|c|c|}
\hline & $\begin{array}{c}\text { Empresas } \\
\text { Nacientes }\end{array}$ & $\begin{array}{c}\text { Empresas } \\
\text { Nuevas }\end{array}$ & $\begin{array}{c}\text { Empresas } \\
\text { Establecidas }\end{array}$ & $\begin{array}{c}\text { PEA } \\
\text { Guayas }\end{array}$ & $\begin{array}{c}\text { TEA } \\
(\%)\end{array}$ & $\begin{array}{c}\text { Empresas } \\
\text { establecidas (\%) }\end{array}$ \\
\hline 2012 & 535 & 4797 & 5050 & 1799221 & 0,296 & 0,281 \\
\hline 2013 & 557 & 5517 & 5488 & 1720708 & 0,353 & 0,319 \\
\hline 2014 & 602 & 6121 & 5773 & 1743443 & 0,386 & 0,331 \\
\hline 2015 & 609 & 6283 & 6364 & 1853859 & 0,372 & 0,343 \\
\hline 2016 & 587 & 6388 & 7397 & 1913954 & 0,364 & 0,386 \\
\hline
\end{tabular}

Fuente: Elaboración propia. Base de datos SRI y Superintendencia de Compañías 
Tabla 7. Tasa de desempleo y VAB de Guayas

\begin{tabular}{|l|c|c|}
\hline & Desempleo & VAB \\
\hline 2012 & $5,5 \%$ & 20439,78 \\
\hline 2013 & $5,4 \%$ & 22763,86 \\
\hline 2014 & $4,6 \%$ & 24749,66 \\
\hline 2015 & $5,2 \%$ & 24409,21 \\
\hline 2016 & $6,4 \%$ & 24970,22 \\
\hline
\end{tabular}

Fuente: Elaboración propia. Base de datos SRI y Superintendencia de Compañías.

La tabla 6 nos indica los porcentajes referentes a la TEA y Empresas Establecidas del estudio, así como el número de Empresas Nacientes, Nuevas, Establecidas y la PEA de Guayas, que fueron empleadas para su cálculo. La TEA de los sectores estratégicos de la provincia en estudio, presenta índices superiores a la Tasa de Empresas Establecidas (Tabla 6), a excepción del año 2016. Con lo que se corrobora lo expuesto en el informe del GEM Ecuador 2016 (2017), donde se menciona que Ecuador se caracteriza por ser un país con altos niveles de emprendimiento, cuya TEA es una de las más altas de la región, y esta a su vez, es superior a la Tasa de Empresas Establecidas, para el caso de la provincia del Guayas. A su vez la tabla 7 nos presenta la evolución del Desempleo y del VAB del Guayas para los años del estudio.

\section{Tabla 8. Matriz de correlaciones}

\begin{tabular}{|l|c|c|c|c|}
\hline & VAB & Desempleo & Establecidas & TEA \\
\hline TEA & 0.9475 & -0.2735 & 0.7014 & 1 \\
\hline Establecidas & 0.8765 & 0.4712 & 1 & \\
\hline Desempleo & -0.0111 & 1 & & \\
\hline VAB & 1 & & & \\
\hline
\end{tabular}

Fuente: Software Gretl. Elaboración propia.

En la tabla 8 se muestran las correlaciones de todas las variables consideradas en el estudio. Se observa que la TEA está fuertemente relacionada con el VAB $(0,9475)$ y las Empresas Establecidas $(0,7014)$. A su vez la correlación entre el VAB y las Empresas Establecidas es positiva $(0,8765)$.

Tabla 9. Contraste de normalidad

\begin{tabular}{|l|c|c|c|}
\hline \multirow{2}{*}{} & \multicolumn{3}{|c|}{ Shapiro-Wilk } \\
\cline { 2 - 4 } & Estadístico & gl & Sig. \\
\hline TEA & 0,859 & 5 & 0,224 \\
\hline Establecidas & 0,984 & 5 & 0,955 \\
\hline Desempleo & 0,955 & 5 & 0,772 \\
\hline VAB & 0,843 & 5 & 0,174 \\
\hline
\end{tabular}

Fuente: Software SPSS. Elaboración propia. 
La tabla 9 nos muestra los resultados de la prueba de Shapiro-Wilk, la cual nos permite estimar si las variables presentan una distribución normal. El estudio se realizó para las diferentes variables de estudio. En todos los casos se puede apreciar que el valor p es superior al $5 \%$ lo que determina que los datos de cada una de las variables siguen una distribución normal.

La tabla 10 recoge los resultados del primer modelo de regresión donde se mide el impacto de la TEA y la Tasa de Empresas Establecidas en el crecimiento económico. Mientras que la tabla 11 muestra los resultados del segundo modelo econométrico donde se mide el impacto de la Tasa de Desempleo y la Tasa de Empresas Establecidas en la TEA.

\section{Tabla 10. Crecimiento económico en función de la TEA y la Tasa de Empresas Establecidas}

\begin{tabular}{|l|c|c|c|c|c|}
\hline & Coeficiente & Desv. Típica & Estadístico t & Valor p & \\
\hline Const & 14.7465 & 0.404546 & 36.4520 & 0.0008 & $* * *$ \\
\hline l_TEA & 0.526539 & 0.105696 & 4.9816 & 0.0380 & $* *$ \\
\hline l_Establecidas & 0.29977 & 0.0934246 & 3.2087 & 0.0849 & $*$ \\
\hline R-cuadrado corregido & 0.970754 & & & \\
\hline Valor p (de F) & 0.014623 & & & \\
\hline $\begin{array}{l}\text { Contraste de normalidad de los residuos: } \\
\text { Hipótesis nula: los errores se distribuyen normalmente } \\
\text { Contraste de Jarque-Bera }=1.22017 \text {, con valor p }=0.543306\end{array}$ \\
\hline
\end{tabular}

Fuente: Software Gretl. Elaboración propia.

La tabla 10 indica que la variable TEA presenta un valor de probabilidad inferior al nivel de significación estadística del 5\% (0,0380), demostrando una relación de incidencia de esta variable sobre el VAB. La relación entre estas variables es directamente proporcional, por cada punto porcentual que se incremente la TEA, el VAB aumentará 0,52 puntos porcentuales. También se refleja que el valor de probabilidad del estimador descriptor de la variable Tasa de Empresas Establecidas, se encuentra cercano al nivel de significación del 5\% (0,0849).

Además, se puede observar que la relación entre la Tasa de Empresas Establecidas y el VAB es directamente proporcional, por cada punto porcentual que se incremente la Tasa de Empresas Establecidas, el VAB aumentará en 0,30 puntos porcentuales.

El valor p del estadístico de Fisher registra un valor inferior al 5\% (0,01462), es decir, se determina que tanto la TEA como la Tasa de Empresas Establecidas inciden en conjunto sobre el VAB. El coeficiente de determinación es de 0,9707 lo que muestra que la TEA y la Tasa de Empresas Establecidas explican en un 97,07\% al VAB. El modelo de regresión logarítmico es idóneo, pues, presenta una relación lineal y sus residuos se encuentran normalmente distribuidos. No se realizó un contraste de no linealidad, debido a que el modelo es logarítmico. El test de normalidad de los residuos indica un valor p no significativo, siendo superior al 5\% (0,5433), lo 
que determina que los errores siguen una distribución normal; es decir que, a pesar de que el número de observaciones sea reducido los estimadores son confiables para hacer predicciones y comprobar hipótesis de relación.

\section{Tabla 11. TEA en función de la Tasa de Desempleo y la Tasa de Empresas Establecidas}

\begin{tabular}{|l|c|c|c|c|c|}
\hline & Coeficiente & Desv. Típica & Estadístico t & Valor p & \\
\hline Const & 0.00267501 & 0.000486464 & 5.4989 & 0.0315 & $* *$ \\
\hline Desempleo & -0.0425209 & 0.00866271 & -4.9085 & 0.0391 & $* *$ \\
\hline Establecidas & 0.95748 & 0.14191 & 6.7471 & 0.0213 & $* *$ \\
\hline R-cuadrado corregido & 0.922128 & & & \\
\hline Valor p (de F) & 0.038936 & & & \\
\hline Contraste de no linealidad (logaritmos) - & & \\
\hline Hipótesis nula: la relación es lineal \\
\hline con valor p = 0.082085 \\
\hline $\begin{array}{l}\text { Contraste de normalidad de los Residuos: } \\
\text { Hipótesis nula: los errores se distribuyen normalmente } \\
\text { Contraste de Jarque-Bera = 1.41296, con valor p }=0.493378\end{array}$ \\
\hline
\end{tabular}

Fuente: Software Gretl. Elaboración propia.

En la tabla 11 se aprecia que el valor de probabilidad del parámetro correspondiente a la variable independiente desempleo es inferior al nivel de significación estadística del 5\% (0,0391), reflejando una relación de incidencia de esta variable sobre la TEA. Se puede observar que la relación entre la TEA y el desempleo es inversamente proporcional debido al signo negativo del estimador, lo que significa que a menor desempleo la TEA aumentará en 0,04 puntos porcentuales. También se identificó que el valor de probabilidad del estimador descriptor de la variable Tasa de Empresas Establecidas es inferior al nivel de significación del 5\% (0,0213), lo que quiere decir que este indicador incide en la TEA. La relación entre estas variables es directamente proporcional; por cada punto porcentual que se incrementa la Tasa de Empresas Establecidas la TEA aumentará en 0,95 puntos porcentuales. El valor p del estadístico de Fisher registra un valor inferior al 5\% (0,03894), por lo tanto, se determina que tanto el desempleo como la Tasa de Empresas Establecidas inciden en conjunto sobre la TEA. El Coeficiente de Determinación fue de 0,9221, lo que muestra que el desempleo y la Tasa de Empresas Establecidas explican en un 92,21\% a la TEA.

El modelo de regresión lineal es idóneo dado que presenta una relación lineal y sus residuos se encuentran normalmente distribuidos. El contraste de no linealidad presentó un valor p superior al nivel de significación estadística del 5\% (0,0821), asumiendo que la relación entre el desempleo, la Tasa de Empresas Establecidas y la TEA presentan una relación lineal; es decir que, no se requiere estimar logaritmos para efectuar la regresión. El test de normalidad de los residuos mostró un valor p no significativo, siendo superior al 5\% (0,4933), lo que determina que los errores siguen 
una distribución normal; es decir que, a pesar de que la muestra sea pequeña los estimadores serán confiables para hacer predicciones y comprobar hipótesis de relación.

\section{Conclusiones y discusión}

Los resultados obtenidos en la tabla 10, en la cual se establece la relación e incidencia positiva de la TEA y la Tasa de Empresas Establecidas en el crecimiento económico, medido por el VAB, concuerda con el estudio de Lupiáñez, Priede y López (2014) en el cual concluyen que existe una relación positiva entre la activada emprendedora y el crecimiento económico, afirmando al emprendimiento como motor del crecimiento económico. De igual forma se corrobora, con la presente investigación, el resultado de Galindo y Méndez (2012), donde los emprendedores ejercen un efecto positivo sobre el crecimiento económico, medido por el PIB, cabe destacar que en las regresiones utilizadas por estos autores se añadió una variable referente a la innovación que también explica positivamente el crecimiento económico, conjuntamente con la actividad emprendedora. Asimismo los resultados presentados en la Tabla 10, compaginan con la investigación de Stam, Suddle, Hessels y Stel (2006); Acs, Audretsch, Braunerhjelm y Carlsson (2012) en la que se determina que la actividad emprendedora tiene un impacto positivo y sistemático en el crecimiento económico, medido por la variación del PIB per cápita. No ocurre lo mismo con los resultados de Jaén, Fernández y Liñán (2013) los cuales muestran una relación inversa de la TEA hacia el crecimiento económico.

En la tabla 11 se evidencia que la TEA está explicada por la Tasa de Desempleo y por la Tasa de Empresas Establecidas, por otro lado, Zuniga et al. (2015) hallaron evidencia de esta relación para un año de estudio donde demostraron que cambios en el desempleo tienen un impacto positivo en el emprendimiento, esto es lo que se conoce, según estos autores, como efecto refugio; es decir, un emprendimiento que nace de la necesidad, donde el desempleo tiene una relación directamente proporcional con el emprendimiento.

En definitiva, las variables desempleo y TEA presentan relación. La disparidad de resultados en los diferentes estudios se presenta en el tipo de relación, es decir, en algunas investigaciones la correlación es positiva y en otras es negativa. En el presente estudio se identificó una relación inversa entre estas variables, lo que indica que cada unidad de desempleo que disminuya influye en un incremento de aproximadamente 4\% de la TEA. En otras palabras, una reducción en el desempleo genera un aumento de la actividad emprendedora. En el presente estudio el autoempleo medido por la TEA significa un aumento del empleo en la región relacionado con una época de expansión de la economía ecuatoriana, registrado en gran parte del período de estudio utilizado, y da cuenta del nacimiento de emprendimientos por oportunidad de mejora y no por una necesidad de empleo. Inclusive se podría pensar que personas que estando empleadas, han decidido emprender como respuesta a oportunidades de mercado o al hecho de disponer de las capacidades necesarias para hacerlo (Pinillos Costa, 2011) o como lo llaman Zuniga, Soza, y Soria (2015), se evidencia el efecto oportunidad.

Esta situación se complementa con la relación positiva encontrada con la variable empresas establecidas y TEA, es decir, el número de empresas establecidas 
aumenta en el ciclo económico expansivo dando lugar al fortalecimiento de clusters empresariales y de un ecosistema emprendedor saludable que da cabida a emprendimientos por oportunidad.

Existe especialización sectorial (clusters) en la provincia del Guayas en Ecuador, específicamente de los sectores textil y confecciones y alimentos y bebidas procesadas, lo cual se evidencia por las altas tasas de entrada de empresas nacientes y nuevas, así como por la tasa de empresas titulares o establecidas. Los sectores estratégicos tradicionales como el astillero, siderurgia y metalmecánica mantienen un aporte de empresas nuevas y nacientes al entramado económico de la provincia y los sectores de desarrollo de software y hardware; y farmacéutica en menor medida.

La contracción de la economía fue detectada por las nuevas y nacientes entradas de los distintos sectores estratégicos identificándose altos índices de turbulencia en el año 2014.

La relación inversa entre el desempleo y el emprendimiento temprano identifica que las empresas creadas en los sectores estratégicos del Guayas surgirían por la oportunidad de mejora.

Finalmente, la evidencia que se presenta ratifica la idea de que el emprendimiento temprano y las empresas establecidas impulsan el crecimiento económico de la provincia del Guayas, como un todo armónico que propicia lo nuevo y mantiene lo existente, de modo que convierte a esta provincia en un polo de desarrollo económico del Ecuador.

\section{Referencias}

Acs, Z. J., Audretsch, D. B., Braunerhjelm, P., \& Carlsson, B. (2012). Growth and entrepreneurship. Small Business Economics, 39(2), 289-300. https://doi.org/10.1007/s11187-010-9307-2

Araque, W. (2015). Emprendimiento en Ecuador. Core Bussiness Ekos. Recuperado de: https:// bit.ly/2OsagkH

Banco Mundial (2014). El emprendimiento en América Latina: Muchas empresas y poca innovación. Banco Mundial. https://doi.org/10.1596/978-1-4648-0284-3

BCE (2017). Valor Agregado Bruto provincial. Cuentas Provinciales Serie 2007-2016. Recuperado de https://www.bce.fin.ec/index.php/component/k2/item/293-cuentas-provinciales/

Dau, L. A., \& Cuervo-Cazurra, A. (2014). To formalize or not to formalize: Entrepreneurship and pro-market institutions. Journal of Business Venturing, 29(5), 668-686. https://doi. org/10.1016/j.jbusvent.2014.05.002

Galindo Martín, M. Á., \& Méndez Picazo, M. T. (2012). Factores que estimulan el emprendimiento y el crecimiento económico. Recuperado de: https://bit.ly/397FJjU

Galindo Martín, M. Á., Méndez Picazo, M. T., \& Castaño Martínez, M. S. (2016). Crecimiento, progreso económico y emprendimiento. Journal of Innovation \& Knowledge, 1(1), 62-68. https://doi.org/10.1016/j.jik.2016.01.006

GEM (2016). Global Entrepreneurship Monitor. GEM América Latina y el Caribe 2015/16. Recuperado de: https://bit.ly/3bcXb8s

GEM (2017). Global Entrepreneurship Monitor. GEM Ecuador 2016. Recuperado de: https://bit. ly/2RZGQN8

Jaén, I., Fernández, J., \& Liñán, F. (2013). Valores culturales, nivel de ingresos y actividad emprendedora. Revista de Economía Mundial, 35(1576-0162), 21-45. Recuperado de: https://bit.ly/2UmSDGM 
INEC (2015). Instituto Nacional de Estadística y Censos. Proyecciones poblacionales del Ecuador, a partir del VII censo de población y VI de Vivienda 2010, actualizadas a la División Político Administrativa 2015. Recuperado de: https://bit.ly/38j1AFk

INEC (2017). Instituto Nacional de Estadística y Censos. Indicadores del Mercado Laboral. Recuperado de: https://bit.ly/38rl6Q5

Liñán, F., Fernández, J., \& Romero, I. (2013). Necessity and Opportunity Entrepreneurship: The mediating of culture. Revista de Economía Mundial, 17(enero), 21-47. https://doi. org/10.3167/sa.2014.580207

Lupiáñez Carrillo, L., Priede Bergamini, T., \& López Cózar, C. (2014). El emprendimiento como motor del crecimiento económico. Boletín económico de ICE, 10. Recuperado de: https:// bit.ly/3bs 4 lpB

Pinillos Costa, M. J. (2011). Cultura postmaterialista y variaciones en el espíritu emprendedor. Investigaciones Europeas de Dirección y Economía de la Empresa, 17(1), 37-55. https:// doi.org/10.1016/S1135-2523(12)60043-4

Rocha, H. (2013). Entrepreneurship and Regional Development: The Role of Clusters. New York: Palgrave and Macmillan.

Rodríguez, A. (2014). Emprendimiento: una megatendencia a nivel mundial. Forbes México. Recuperado de: https://bit.ly/2HdSH3I

SENPLADES (2015). Secretaría Nacional de Planificación y Desarrollo. Agenda Zonal, Zona 8 Guayaquil. Recuperado de: https://bit.ly/2UMpjtD

SRI (2018). Servicio de rentas internas. Base de datos del Registro Único de Contribuyentes. Recuperado de: https://bit.ly/2uC57jb

Stam, E., Suddle, K., Hessels, S. J. A., \& Stel, A. van. (2006). Los emprendedores con potencial de crecimiento y el desarrollo económico . Políticas públicas de apoyo a los emprendedores. Ekonomiaz, 62, 1-26. Recuperado de: https://bit.ly/37hBe4Y

SUPERCIAS (2018). Superintendencia de compañías, valores y seguros. Directorio de compañías. Recuperado de: https://bit.ly/3brEz4K

Zuniga Jara, S., Soza Amigo, S., \& Soria Barreto, K. (2014). Dinámica del emprendimiento y el desempleo en Guatemala. Pensamiento \& Gestión, 43(1), 103-115. https://doi.org/10.4067/s 0718-22442015000100007 